\title{
Température corporelle des Zébus et des métis Zébus taurins sous les tropiques
}

\author{
par J. PAGOT
}

\section{INTRODUCTION}

Chez tous les animaux à sang chaud, la température corporelle reste constante entre certaines limites. L'animal, par un processus physiologique, maintient un équilibre entre la chaleur de son métabolisme, la chaleur qu'il reçoit éventuellement de l'ambiance et celle qu'il émet, suivant le schéma ci-dessous :

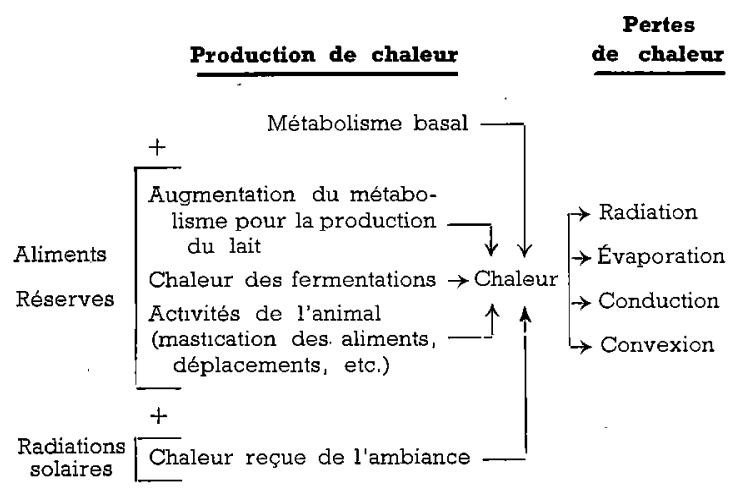

Si la production de chaleur est influencée par des facteurs tels que l'alimentation, l'activité, la production laitière, son élimination dépend surtout des conditions de l'ambiance et, en particulier, du climat, qui conditionne l'efficacité des facteurs physiques de la régulation thermique puisque, dans certains cas, l'homéotherme doit éliminer, en plus de la chaleur dégradée de son métabolisme, la chaleur qu'il reçoit de l'ambiance.

Max Sorre a classé les climats en fonction du rôle que doivent remplir les mécanismes physiologiques pour maintenir la constance de la température corporelle. Il distingue :

10 Des climats à thermogenèse ou climats froids, où les mécanismes régulateurs ont à faire face à une forte déperdition de chaleur et doivent donc produire de la chaleur;

$2^{\circ}$ Des climats à thermolyse ou climats chauds, où les mécanismes régulateurs doivent éliminer la chaleur du métabolisme et celle reçue de l'ambiance;

$3^{\circ}$ Les climats tempérés, dans lesquels l'équilibre thermique est aisément assuré et où la régulation se fait alternativement par thermolyse ou thermogenèse.

Dans notre étude, nous avons essayé de déterminer dans quelle mesure les facteurs météorologiques d'un " climat à thermolyse » étaient susceptibles d'influencer la température corporelle d'un groupe de zébus et de leur métis avec des, races européennes charollaise et montbéliarde.

\section{I. - PROTOCOLE EXPÉRIMENTAL}

\section{A. - Animaux utilisés :}

Les observations ont été faites sur. 20 vaches adultes réparties en quatre lots :

ler Vaches pur-sang zébu peuhl.

$2^{\text {e }}$ Vaches demi-sang zébu-Charollais.

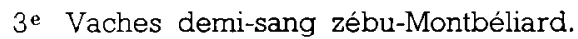

$4^{\mathrm{e}}$ Vache quart-sang zébu-Montbéliard (rétrocroisement de vaches peuhles par un taureau demisang Montbéliard).

La robe des zébus était pie-rouge ou pie-noire; celle des demi-Charollais était froment clair; cello des demi-Montbéliard et des quart-Montbéliard pie-rouge.

Les plages blanches étaient assez étendues sur la robe des demi-Montbéliard et réduites sur celle des quart-Montbéliard.

Les caractéristiques métriques moyennes des quatre groupes d'animaux étaient les suivantes : 


\begin{tabular}{|c|c|c|c|c|}
\hline & $\begin{array}{c}1 / 2 \\
\text { CHAROLLAIS }\end{array}$ & $\begin{array}{c}1 / 2 \\
\text { MONTBÉLIARD }\end{array}$ & $\begin{array}{c}1 / 4 \\
\text { MONTBÉLIARD }\end{array}$ & ZÉBUS \\
\hline Poids moyon,$\ldots \ldots \ldots \ldots \ldots \ldots \ldots$ & $388 \mathrm{~kg}$ & $334 \mathrm{~kg}$ & $245 \mathrm{~kg}$ & $230 \mathrm{~kg}$ \\
\hline Taille au garrot. . & $127 \mathrm{~cm}$ & $126 \mathrm{~cm}$ & $119 \mathrm{~cm}$ & $116 \mathrm{~cm}$ \\
\hline Péririètre thuracique... & $169 \mathrm{~cm}$ & $160 \mathrm{~cm}$ & $147 \mathrm{~cm}$ & $144 \mathrm{~cm}$ \\
\hline Largeur de poitrine $\ldots \ldots \ldots \ldots \ldots \ldots \ldots$ & $36 \mathrm{~cm}$ & $31 \mathrm{~cm}$ & $29 \mathrm{~cm}$ & $28 \mathrm{~cm}$ \\
\hline Hauteur de poitrine.. & $57 \mathrm{~cm}$ & $65 \mathrm{~cm}$ & $60 \mathrm{~cm}$ & $59 \mathrm{~cm}$ \\
\hline $\begin{array}{l}\text { Distance pointe de l'épaule-pointe de la } \\
\text { hanche } \ldots \ldots \ldots \ldots \ldots \ldots \ldots \ldots \ldots \ldots \ldots\end{array}$ & $109 \mathrm{~cm}$ & $104 \mathrm{~cm}$ & $93 \mathrm{~cm}$ & 94 CnI \\
\hline Longueur du bassin. & $57 \mathrm{~cm}$ & $45 \mathrm{~cm}$ & $43 \mathrm{~cm}$ & $41 \mathrm{~cm}$ \\
\hline Largeur du bassin & $49 \mathrm{~cm}$ & $48 \mathrm{~cm}$ & $41 \mathrm{~cm}$ & $42 \mathrm{~cm}$ \\
\hline
\end{tabular}

Les vaches en lactation ont donné en moyenne en une lactation de 300 jours :

$1 / 2$ Charollais . . . . . . . . . . . $1.598 \mathrm{~kg} \pm 56$

$1 / 2$ Montbéliard . . . . . . . . . . . . $1.697 \mathrm{~kg} \pm 50$

$1 / 1$ Montbéliard . . . . . . . . . . . . . $1.047 \mathrm{~kg} \pm 60$

Zébu pur sang.............. $1.041 \mathrm{~kg} \pm 62$

\section{B. - Techniques d'observations :}

La température corporelle a été prise chaque jour pendant un an, le matin à $7 \mathrm{~h}$ 30, avant le départ au pâturage, et le soir vers 18 heures, une demiheure après le retour à l'étable.

Une station de météorologie voisine de la ferme relevait la température et l'humidité ambiantes.

On a fait également des observations suivant le protocole de Rhoad en vue de déterminer, pour chaque groupe, le coefficient d'adaptabilité $A$ de cet auteur.

$$
A=100-10(B T-101.0)
$$

où $\mathrm{BT}$, exprimé en degrés Fahrenheit, désigne la moyenne des températures corporelles prises à 10 heures et 15 heures.

\section{C. ..- Présentation des résultats :}

On a calculé les moyennes pour chaque groupe d'animaux en utilisant tous les chiffres enregistrés au cours de l'année.

L'approximation indiquée pour les moyennes a été calculée par la formule :

$$
\mathrm{e}=1,96 . \sigma \bar{x}
$$

$\sigma_{X}$ étant l'écart type de la moyemne calculée;

$\mathrm{Ta}$ représente la température ambiante; elle est exprimée en degrés centigrades;

$\mathrm{Tu}$ la tension de vapeur; elle est exprimée en millibars;
U l'humidité relative ; elle est exprimée en degrés hygrométriques;

Tc la température corporelle; elle est exprimée en degrés centigrades.

\section{RÉSULTATS}

\section{I. - Caractéristiques du climat}

Le climat de la station de Segou est du type tropical. Le tableau $n^{\circ} 1$ donne les caractéristiques du climat de l'année 1950.

\section{A. - Température.}

Les températures moyennes mensuelles maxima sont enregistrées en mars, avril et mai : $37^{\circ} 2,39 \circ 8$, 3903 , et, les températures moyennes mensuelles minima en décembre, janvier et février : 1505 , $15^{\circ}, 17^{\circ}$.

Les températures maxima sont enregistrées en avril et mai : $42^{\circ} 7,41^{\circ} 8$; les températures minima en novembre et décembre : $11^{\circ} 0,11^{\circ} 6$.

La courbe de la température mensuelle moyenne du matin (tableau $n^{\circ}$ 2) présente un minima en décembre : 1703, un maxima en mai : $27^{\circ} 1$; les variations d'un mois au suivant sont régulièrement croissantes de janvier à mai et régulièrement décroissantes de mai à décembré.

La courbe de la température mensuelle moyenne du soir est moins régulière que celle de la température du matin; 'elle présente deux minima : en décembre $28^{\circ} 5$, en août $27^{\circ} 2$, et deux maxima : en mai $37^{\circ} 5$ et en octobre $29^{\circ} 5$.

La distribution des températures du matin et du soir autour de la moyenne annuelle pouvant être 
assirnilée à une distribution normale, on a calculé les températures moyennes annuelles :

Matin .................... 22060 \pm 0.22

Soir . . . . . . . . . . . . . . . . . 31094 \pm 0052

B. - Précipitations.

Les précipitations maxima ont lieu en juillet et août : $218,5 \mathrm{~mm}$.

Au cours de la saison des pluies 1950, il est tombé $899,1 \mathrm{~mm}$ en 68 jours.

Au cours des années précédentes, il est tombé en moyenne $656,6 \mathrm{~mm}$.

\section{C. - Tension de vapeur (tableau no 3 ).}

Ia tension de vapeur du matin est minima en janvier et février : $9,3 \mathrm{mb}$ et $8,2 \mathrm{mb}$ et maxima en août, septembre et octobre : $28,0 \mathrm{mb}, 27,4 \mathrm{mb}$ et $26,9 \mathrm{mb}$.

La tension de vapeur du matin présente un minimum en février : $8,2 \mathrm{mb}$, et un maximum en septembre : $26,5 \mathrm{mb}$. La courbe des variations d'un mois au suivant est régulièrement croissante de février à septembre et régulièrement décroissante d'octobre à février.

La tension de vapeur du soir présente un minimum en février : 7,2 mb, un maximum en août : $28,0 \mathrm{mb}$. La courbe des variations d'un mois au suivant est régulièrement croissante de février à août et régulièrement décroissante de septembre à février.

\section{D. - Évaporation.}

L'évaporation en 24 heures est minima en août, septembre et octobre : $74,0-86,6-118,3 \mathrm{~mm}$, et maxima en février, mars et avril : 345,6 - 479,9 $484,1 \mathrm{~mm}$.

\section{E. - Humidité relative (tableau no 2).}

L'humidité relative du matin est minima en février et mars : 38 et $30 \%$, et maxima en août, septembre et octobre : 93,93 et $90 \%$.

L'humidité relative du soir est minima en février et mars : $17,14 \%$, et maxima, en août, septembre et octobre : 78,74 et $65 \%$.

Les courbes de l'humidité relative se superposent à celles de la tension de vapeur.

La distribution des valeurs journalières du matin et du soir de la tension de vapeur et de l'humidité relative n'est pas normale, ce qui rend impossible le calcul de moyennes annuelles significatives.

Les courbes de distribution sont bi-modales, chaque courbe élémentaire correspondant l'une à la saison sèche, l'autre à la saison des pluies.

Ainsi, l'année peut être divisée en deux saisons : une de faible tension de vapeur correspondant aux mois de décembre, janvier, février, mars, avril et mai; une de forte tension de vapeur : juillet, août, septembre et octobre.

$\mathrm{Au}$ cours des mois de juin et de novembre, qui sont les saisons intermédiaires, la tension de vapeur et l'humidité relative présentent d'un jour au suivant des écarts très grands de l'ordre de $15 \mathrm{mb}$ et $20 \%$ respectivement.

\section{F. - Climatogrammes.}

Wright, Dorno et Azzi ont indiqué une technique permettant de représenter par une courbe les deux caractéristiques principales du climat : température et humidité relative.

On porte en abscisse l'humidité relative moyenne mensuelle et en ordonnée la température moyenne mensuelle; on joint les points obtenus par ordre chronologique (tableau no 4).

Nous avons tracé de même un climatogramme en portant en abscisse la tension de vapeur et en ordonnée la température ambiante (tableau no 5 ).

Ces courbes donnent une représentation graphique des variations concomitantes de la température ambiante et de l'état hygrométrique de l'atmosphère.

\section{II. - Températures corporelles des groupes d'animaux.}

\section{A. - Températures moyennes annuelles (tableaux 6 et 7 ).}

Les températures journalières $\mathrm{du}$, matin et $\mathrm{du}$ soir se répartissent normalement autour de la moyenne. Utilisant toutes les températures enregistrées, nous avons calculé ces moyennes :

\section{Matin :}

\begin{tabular}{|c|c|}
\hline $1 / 2$ Charollais & $38^{\circ} 19 \pm 0.01$ \\
\hline I/2 Montbéliard ...... & $38^{\circ} 17 \pm 0.01$ \\
\hline 1/4 Montbéliard ...... . & $38^{\circ} 14 \pm 0001$ \\
\hline Zébu .......... & $38^{\circ} 15 \pm 0.009$ \\
\hline Soir : & \\
\hline $1 / 2$ Charollais & $38.93 \pm 0.04$ \\
\hline I 2 Montbéliard ....... & $38 \circ 83+0002$ \\
\hline $1 / 4$ Montbéliard ............. & $38^{\circ} 83 \pm 0.03$ \\
\hline Zébu $\ldots \ldots \ldots \ldots \ldots \ldots \ldots \ldots$ & $38^{\circ} 84 \pm 0003$ \\
\hline
\end{tabular}

Les différences entre les températures moyennes annuelles du matin prises deux à deux sont significatives, sauf pour le couple 1/4 Montbéliard-zébu.

Pour les observations du soir, seule la température moyenne annuelle des $1 / 2$ Charollais est significativement différente des trois autres.

Les écarts entre les températures moyennes 
annucllos du matin of du soir de chacun des groupes d'animaux sont les suivants :
$1 / 2$ Charollais
$0074+0,05$
$1 / 2$ Montbéliard.
$0 \circ 66 \pm 0,03$
$1 / 4$ Montbéliard.
$0^{\circ} 69 \pm 0,04$
Zébus.
$0,69 \pm 0,04$

Seules, les différences entre la valeur de l'écart pour los $1 / 2$ Charollais et celles des autres groupes sont significatives.

\section{B. - Étude des courbes des températures} moyennes mensuelles (tableaux 8 à 11).

Au cours del'année, les courbes des températures matinales et vespérales moyennes mensuelles présentent un cycle.

Les courbes des quatre groupes d'animaux sont semblables; les maxima et les minima sont enregistrés aux mêmes époques de l'année.

Courbes du matin. - Les courbes du matin sont très aplaties; l'amplitude des variations au cours de l'année par catégorie d'animaux est la suivante :

Différence

\begin{tabular}{|c|c|c|}
\hline Zébus & $38^{\circ} 04$ à $38^{\circ} 19$ & $0^{\circ} 15$ \\
\hline 1/4 Montbéliard... & $38^{\circ} 05$ à $38^{\circ} 28$ & 0023 \\
\hline Tonthóliard & $38 \circ 04$ à $38 \circ 26$ & 020 \\
\hline 1/2 Charollais. . & $38^{\circ} 08^{2}$ à $38^{\circ} 27$ & \\
\hline
\end{tabular}

Les courbes des quatre groupes présentent chacune deux clochers : l'un, le plus élevé, de mars à mai, l'autre, moins élevé, en septembreoctobre.

Les groupes d'animaux se classent dans l'ordre décroissant des valeurs des températures moyennes mensuelles maxima de la facon suivante:

\begin{tabular}{|c|c|}
\hline $1 / 4$ Monthéliard ......... & 38028 (mai) \\
\hline 1/2 Charollais... & $38^{\circ} 27$ (juin) \\
\hline 1/2 Montbéliard & 38026 (mars) \\
\hline Zébus ...... . & $38^{\circ} 19$ (mai-juin-juillet) \\
\hline
\end{tabular}

Courbes du soir. - Les courbes des températures moyennes mensuelles du soir ont des amplitudes de variations supérieures à celles du matin :

Différence

Zébus ............ $38^{0055}$ à $39^{\circ} 16 \quad 0^{\circ} 61$

$1 / 4$ Montbéliard... . . . . 3 38052 à 39n10 0058

1/2 Monbéliard . . . . . . . 38 3859 à 39004 0045

$1 / 2$ Charollais......... $38^{\circ} 58$ à $39^{\circ} 60 \quad 1$ o02

Les courbes des quatre groupes d'animaux présentent chacune un maxima au cours des mois de septembre, octobre et novembre.

Les groupes d'animaux se classent, dans l'ordre dćcroissant des valeurs des températures mensuelles maxima, de la façon suivante :
$1 / 2$ Charollais $\ldots \ldots \ldots \ldots \ldots$. $39 \circ 53$ (septembre)
Zébus ................ $39 \circ 16$ (octobre)
1/4 Montbéliard ........... 39010 (septembre)
1/2 Montbéliard ............ 39004 (octobre)

C. - Écarts entre les températures moyennes mensuelles du matin et du soir (tableau 12).

Les courbes des écarts entre les températures du matin et du soir des zébus, des $1 / 2$ Montbéliard et des $1 / 4$ Montbéliard sont presque identiques.

Les courbes des écarts des trois premiers groupes présentent des maxima de l'ordre de $0 \circ 9$ en septembre, octobre et novembre, et des minima de 004 à 005 , en février, mars et avril.

La courbe des I/2 Charollais a la même allure générale que les trois autres; elle n'en diffère que par la valeur des maxima : 008 et $1^{\circ} 4$.

Nous avons vu plus haut que seuls, les 1/2 Charollais se distinguaient des trois autres groupes par la valeur de la différence entre les températures moyennes annuelles du matin et du.soir.

\section{III. - Corrélations entre la température corporelle et les caractéristiques du climat.}

\section{A. - Généralités.}

De nombreux auteurs ont essayé de calculer les corrélations existant entre la température ambiante, l'humidité et la température corporelle des mammifères et des oiseaux.

$\mathrm{Si}$, expérimentalement, en chambre climatisée, on peut contrôler les deux variables : température ambiante et tension de vapeur, il n'en est pas de même lorsque les animaux sont entretenus en élevage ouvert et qu'ils sont soumis aux conditions naturelles.

Pour calculer une fonction du type

$$
\mathrm{T} c=f(\mathrm{Ta})+f(\mathrm{~T} u)
$$

on est amené à faire l'analyse statistique des observations et à déterminer d'abord les coefficients de corrélation totale entre les trois caractéristiques et ensuite les coefficients de corrélation partielle entre les variables prises deux à deux, la troisième étant constante.

Cette analyse suppose que les distributions des trois variables isont normales.

Nous avons vu précédemment que, si les distributions de la température ambiante et de la température corporelle étaient normales, il n'en était pas de même des distributions de la tension de vapeur et de l'humidité relative.

Il n'est donc pas possible de calculer les coefficients de corrélation totale entre la température 
corporelle et la tension de vapeur ou l'hurnidité, et, a fortiori, les coefficients de corrélation partielle.

Les travaux qui peuvent servir de référence pour ceux dans lesquels les auteurs ont appliqué la technique d'analyse des corrélations multiples sont ceux de Seath et Miller.

Ils prennent à 15 heures, deux jours par semaine, la température corporelle de vaches jersiaises et de vaches Holstein, une première année du 28 août au 14 septembre, une seconde année, 15 fois entre le 16 juillet et le 3 août pour un troupeau et entre le 6 et le 24 août pour l'autre troupeau.

Ils ont trouvé les équations suivantes dans lesquelles la température corporelle (Tc) est une normales et, par là, leur a permis de calculer des coefficients de corrélation partielle; cependant, eux-mèmes remarquent qu'au cours de la première année, la corrélation entre la température ambiante et l'humidité était positive et qu'elle était négative au cours de la seconde année.

\section{B. - Recherches personnelles.}

En utilisant toutes les températures corporelles enregistrées le matin et le soir, nous avons constaté, en calculant les coefficients de corrélation totale, température ambiante, température corporelle :

a) qu'il était impossible de calculer un coefficient unique pour les températures du matin et celles du

TABLEAU B

\begin{tabular}{|r|c|c|c|c|c|c|}
\hline & \multicolumn{2}{|c|}{1944} & & \multicolumn{2}{|c|}{1945} \\
\cline { 2 - 6 } & Extrêmes & Moyennes & Ecart type & Extrêmes & Moyennes & Écart type \\
\cline { 2 - 6 } & & & & & & \\
Température ambian- & $65-93$ & 85,1 & 6,17 & $75-91$ & 85,6 & 4,75 \\
te (Fahrenheit).... & $27-91$ & 56,8 & 14,06 & $51-91$ & 73,5 & 10,00 \\
\hline
\end{tabular}

fonction linéaire de la température ambiante $\mathrm{T}$ et de l'humidité $\mathrm{H}$ ( $\mathrm{Tc}$ et $\mathrm{T}$ sont exprimées en degrés Fahrenheit, $\mathrm{H}$ en pourcentage).

Temperature corporelle des jersiaises

$\mathrm{I}^{\text {re }}$ année $: \mathrm{TC}=0,1007 \mathrm{~T}+0,00758 \mathrm{H}+93^{\circ} 65$;

$2^{\mathrm{e}}$ année $: \mathrm{T} c=0,1919 \mathrm{~T}-0,0321 \mathrm{H}+88085$.

Température corporelle des Holstein

Ire année $: \mathrm{T} c=0,1768 \mathrm{~T}+0,00636 \mathrm{H}+87^{\circ} 17$;

$2^{\mathrm{e}}$ année $: \mathrm{Tc}=0,1721 \mathrm{~T}-0,00303 \mathrm{H}+88091$.

Les caractéristiques atmosphériques pendant l'expérience étaient celles indiquées au Tableau B.

La faible durée des observations a permis aux auteurs d'obtenir pour les variables des distributions soir, les distributions marginales des. tableaux de corrélation n'étant pas normales;

b) qu'en groupant séparément les observations du matin et du soir, les distributions marginales des tables de corrélation étaient normales;

c) que les coefficients de corrélation totale; température ambiante/température corpor'elle calculés. étaient significatifs pour tous les groupes d'animaux et qu'ils avaient les valeurs indiquées au Tableau C

L'ajustement à des droites est acceptable pour les observations du matin mais ne l'est, pour les observations du soir, que dans la zone des températures ambiantes moyennes.

Nous avons cependant calculé les équations de

TABLEAU C

\begin{tabular}{|c|c|c|}
\hline & $=\begin{array}{l}I_{0,} \mathrm{Ta} \\
\text { Matin }\end{array}$ & $\begin{array}{l}\text { r, Tc, Ta } \\
\text { Soir }\end{array}$ \\
\hline $1 / 2$ Charollais $\ldots \ldots \ldots \ldots \ldots \ldots \ldots \ldots$ & $-0,418 \pm 0,095$ & $-0,395 \pm 0,097$ \\
\hline 1/2 Montbéliard........ & $\div 0,356 \div 0,097$ & $-0,249 \pm 0,008$ \\
\hline 1/4 Montbéliard...................... & $+0,386 \pm 0,09$ & $-0,425 \pm 0,095$ \\
\hline Zébus & $\div 0,403 \pm 0,07$ & $-0,771 \pm 0,406$ \\
\hline
\end{tabular}


régression de la température corporelle sur la température ambiante (Tableau D).

Il est bien entendu que ces équations ne valent que pour les tensions de vapeur observées et ne sont pas susceptibles d'être généralisées.

C. - Analyse graphique des variations de la température en fonction des caractéristiques du climat.

Avec les températures corporelles moyennes mensuelles, les tensions de vapeur moyennes mensuelles, les températures ambiantes moyennes mensuelles, nous avons, utilisant la même technique que pour
En faisant intervenir les valeurs des différentes variables, on voit que :

a) $\mathrm{Si}$ la température ambiante reste constante aux environs de $30^{\circ}$, une diminution de la tension de vapeur de $20 \mathrm{mb}$ entraîne une diminution de la température corporelle de tous les groupes de 5/10 de degré;

b) Si la température ambiante diminue de 3705 à $27^{\circ} 2$, soit d'cnviron 1003, et que la tonsion-de vapour augmente de $15,9 \mathrm{mb}$ à $28 \mathrm{mb}$, soit de $13 \mathrm{mb}$, la température corporelle reste constante à $38^{\circ} 7$;

c) Si la tension de vapeur est élevée, supérieure à $26,9 \mathrm{mb}$, et le degré hygrométrique supérieur

TABLEAU D

\begin{tabular}{|c|c|c|}
\hline & MATIN & SOIR \\
\hline $1 / 2$ Charollais $\ldots \ldots \ldots \ldots \ldots \ldots \ldots \ldots \ldots$ & $\mathrm{Tc}=0,258 \mathrm{Ta}+32^{\circ} 76$ & $\mathrm{Tc}=58009 \longrightarrow 0,600 \mathrm{Ta}$ \\
\hline 1/2 Montbéliard... . . . . . . . . . . & $\mathrm{Tc}=0,216 \mathrm{Ta}+33^{\circ} 20$ & $\mathrm{~T}_{C}=46^{\circ} 01-0,225 \mathrm{Ta}$ \\
\hline 1/4 Montbéliard...................... & $\mathrm{T} c=0,200 \mathrm{Ta}+33^{\circ} 62$ & $\mathrm{Tc}=50^{\circ} 10-0,353 \mathrm{Ta}$ \\
\hline Zébus . & $\mathrm{Tc}=0,148 \mathrm{Ta}+3408 \mathrm{I}$ & $\mathrm{Tc}=63^{\circ} 30-0,766 \mathrm{Ta}$ \\
\hline
\end{tabular}

les climatogrammes, essayé de déterminer l'influence relative de la température corporelle.

Observations du matin : Les polygones sont caractérisés par leur faible surface, la droite de régression de la température corporelle sur la température ambiante donne une idée exacte de la corrélation.

Observations du soir : En mai, juin, juillet et aoüt, la température ambiante diminue, la tension de vapeur augmente; on observe pour tous les groupes d'animaux que la température corporelle reste constante.

En août, septembre et octobre, la température ambiante augmente légèrement; la tension de vapeur reste presque constante mais très forte (supérieure à $26,9 \mathrm{mb}$ ) ; le degré hygrométrique est également très élevé (supérieur à $65 \%$ ); la température corporelle augmente, pour tous les groupes, de plus de 0020.

En octobre, novembre, décembre et janvier, la température ambiante reste pratiquomont constantc, la tension de vapeur diminue de près de $10 \mathrm{mb}$, le pourcentage d'humidité diminue de près de $60 \%$; la température corporelle de tous les groupes diminue.

En février, mars, avril et mai, la température ambiante et la tension de vapeur augmentent, la température corporelle fait de même. à $65 \%$, une très faible variation de la température ambiante de $27^{\circ} 5$ à $29 \circ 5$ entraîne une forte augmentation de la température corporelle.

Cherchant à expliquer ces faits, nous avons été amené à considérer non plus la valeur absolue de la tension de vapeur mais le déficit de saturation égal à la différence entre la tension de vapeur maxima pour la temperature ambiante observee et la tension de vapeur observée, car les mécanismes régulateurs physiologiques de la température faisant intervenir surtout des phénomènes d'évaporation, ils auront à faire face à un travail d'autant plus important que le déficit de saturation sera faible et que la température ambiante sera plus élevée.

\section{IV. - Action du déficit de saturation et de la température ambiante sur la température corporelle.}

\section{A. - Analyse des courbes température ambiante/} déficit de saturation.

Nous avons tracé, à l'aide des chiffres du tableau no 21, les courbes des variations concomitantes du déficit de saturation et de la température ambiante (tableau no 22).

Observations du matin.

En juin, juillet, août, septembre et octobre, le 
déficit de saturation est très faible, inférieur à $5 \mathrm{mb}$, la température à une valeur moyenne.

En novembre, décembre, janvier et février, le déficit de saturation a une valeur moyenne, les températures sont très faibles, inférieures à $20^{\circ}$.

Le mois de mars correspond à un déficit de saturation élevé et à une température ambiante moyenne.

En avril, mai et juin, le déficit de saturation est relativement élevé, la température ambiante est élevée.

\section{Observations du soir.}

L'année se divise de la façon suivante:

10 En juillet, août, septembre et octobre, le déficit de saturation est très faible, inférieur à $14 \mathrm{mb}$. La température ambiante est relativement faible.

$2^{\circ}$ En avril et mai, le déficit de saturation est élevé, supérieur à $48 \mathrm{mb}$. La lempéralure ambiante est voisine de $38^{\circ}$.

$3^{\circ}$ En novembre, décembre, janvier et février, le déficit de saturation a des valeurs moyennes de 13,9 à $36,5 \mathrm{mb}$. La température ambiante est faible.

$4^{\circ}$ En mars et juin, le déficit de saturation a des valeurs moyennes de 33,9 à $38,9 \mathrm{mb}$. La température ambiante est élevée.

\section{B. - Rapport entre la temperature corporelle et le déficit de saturation.}

Dans le calcul du déficit de saturation, on fait intervenir directement la tension de vapeur et indirectement la température ambiante, puisque celle-ci intervient dans le calcul de la tension de vapeur maxima. Ainsi, on peut regarder les courbes des graphiques 23 à 26 , comme les résultantes de l'association des courbes température ambiante: température corporelle, et tension de vapeur,' température corporelle.

L'examen des graphiques 23 à 26 montre que c'est au cours des mois où le déficit de saturation est le plus faible qu'on enregistre les plus fortes températures corporelles et qu'inversement, c'est au cours des mois où le déficit de saturation est maximum que l'on enregistre les températures corporelles minima.

En avril et mai, bien que la température soit élevée, la température corporelle garde une valeur moyenne car le déficit de saturation est élevé.

Le déficit de saturation a donc une influence beaucoup plus grande que la température ambiante.

Cette déduction est confirmée par les résultats que nous avons obtenus dans le calcul des coefficients de corrélation entre la température corporelle et la température ambiante, coefficients qui sont positifs le matin et négatifs le soir.
Le matin, le déficit de saturation est faible, l'augmentation de la température ambiante entraîne une augmentation de la température corporelle, alors que, le soir, malgré une augmentation de la température ambiante, et du fait que le déficit de saturation augmente, la température corporelle diminue, l'avantage résultant de l'augmentation' du déficit de saturation étant supérieur à l'action de l'augmentation de la température ambiante.

\section{C. - Zones de confort thermique.}

Si l'on compare les courbes de température des différentes catégories d'animaux, on voit que le matin, c'est au cours des mois de novembre, décembre, janvier et février que les températures corporelles les plus basses sont enregistrées et que le soir, c'est en janvier, février et mars qu'on enregistre les températures corporelles les plus faibles.

En joignant, sur le graphique 22, les points caractéristiques de novembre le matin, à janvier le soir, et janvier le matin à mars le soir, on délimite une zone de température et d'humidité qu'on peut considérer comme étant celle qui correspond au confort maximum pour le climal consideré.

Les équations des deux droites limitant cette zone. sont les suivantes :

$$
\begin{aligned}
& \mathrm{T} a=0,438 . \Delta \mathrm{T} u+16,63 \\
& \mathrm{~T} a=0,452 . \Delta \mathrm{T} u+12,70
\end{aligned}
$$

Pour une température ambiante considérée, l'ambiance sera confortable si le déficit de saturation exprimé en millibars satisfait à la: double inégalité suivante :

$$
\frac{\mathrm{T} a-12,70}{0,452}>\mathrm{T} u \max -\mathrm{T} u>\frac{\mathrm{T} a-\mathrm{I} 6,63}{0,458}
$$

Pour un déficit de saturation donné, les températures extrêmes de la zone de confort différeront de :

$$
\mathrm{Ta}=3093-0,014 \Delta \mathrm{T} u
$$

Pour un déficit de saturation doriné, l'ambiance sera confortable si la température ambiante satisfait à la double inégalité suivante :

$$
0,438 \perp \mathrm{T} u+16,63>\mathrm{T} a>0,452 \Delta \mathrm{T} u+12,70
$$

Pour une temperature ambiante donnée, les déficits de saturation extrêmes de la zone de confort différeront de :

$$
\Delta \mathrm{T} u=9,87 \mathrm{mb}-0,07 \mathrm{Ta}
$$

Il est remarquable que la zone de confort pour un déficit de saturation nul coupe l'axe des températures entre les températures de $12^{\circ} 7$ et $16^{\circ} 6$, c'est-à-dire autour du point $15^{\circ}$. 


\section{v. - Comparaison des réactions des différents groupes d'ànimaux.}

A. - Comparaisons des réponses des zébus à celles des métis zébus-Montbéliard :

10 Les températures corporelles.

Les températures corporelles moyennes annuelles du matin sont pratiquemont identiques chez les zébus et les 1/4 Montbéliard.

Les températures moyennes annuelles du soir sont pratiquement identiques pour les trois groupes.

L'amplitude des variations des températures moyennes mensuelles du matin est très faible chez les zébus; elle a une même valeur, supérieure à celle observée chez les zébus, pour les $1 / 4$ et 1/2 Montbeliard.

L'amplitude des variations des températures moyennes mensuelles du soir des 1/4 Montbéliard a une valeur intermédiaire, les zébus faisant la plus forte amplitude de variations.

Les températures moyennes maxima du matin sont pratiquement identiques pour les $1 / 4$ et $1 / 2$ Montbéliard, leurs valeurs sont supérieures à celles des zébus.

La température moyenne maxima du soir des $1 / 4$ Montbéliard est intermédiaire entre celle des $1 / 2$ Montbéliard et celle des zébus qui est la plus élevée.

Il semblerait que, pour les températures basses, les 1/4 Montbéliard se comportent comme les 1/2 Montbéliard et que, pour les températures élevées, la différence génétique due au sang zébu interviendrait.

$\mathrm{Si}$ on compare indintenant les températures moyennes mensuelles minimales du matin et maximales du soir, on note que ce sont les zébus qui présentent les valeurs extrêmes, ce qui indique que leur température corporelle varie dans de plus grandes limites que celles des autres groupes.

Cette conclusion est confirmée par l'examen des courbes températures corporelles/température ambiante, température corporelle/tension de vapeur.

$2^{\circ}$ Courbes température ambiante/température corporelle.

Les courbes obtenues pour les différents groupes d'animaux sont semblables, elles ne diffèrent que par leur surface.

Les courbes établies avec les observations du matin sont très ramassécs; la surfaco do cello des zébus est très faible, celle des $1 / 2$ sang est la plus grande, celle des $1 / 4$ sang est intermédiaire.

Les courbes obtenues avec les observations du soir se disposent dans un ordre inverse, la surface de celle des zébus est plus grande, celle des $1 / 2$ Montbéliard est la moins étendue, celle des I/4 Mont- béliard est intermédiaire, mais très proche de celle des zébus.

$3^{\circ}$ Courbes tension de vapeur/température corporelle.

Seule parmi les courbes établies avec les observations du matin, celle des 1/2 Montbéliard a une surface plus grande que celle des deux autres groupes.

De même que pour les observations du matin, les courbes du soir des zébus et des 1/4 Montbéliard sont pratiquement identiques, mais la surface de la courbe des 1/2 Montbéliard est inférieure à celle des deux autres groupes.

$4^{\circ}$ Courbes déficit de saturation/température corporelle.

Les courbes des tableaux 23 à 26 confirment les remarques faites à propos des autres courbes. Les courbes des trois groupes sont semblables et ne diffèrent que par leur surface.

B. - Comparaison des réponses des 1/2 Charollais à celles des $1 / 2$ Montbéliard.

Par rapport aux 1/2 Montbéliard, les 1/2 Charollais semblent déséquilibrés, leurs réponses aux variations de la température, de l'humidité ambiante et du déficit de saturation sont beaucoup plus fortes.

Plus généralement, les $1 / 2$ Charollais sont en hyperthermie par rapport aux autres groupes.

\section{vI. - Conclusions.}

En comparant entre elles les courbes des différentes catégories d'animaux, on peut conclure que les $1 / 2$ Charollais ont une température corporelle qui varie dans de grandes limites et qu'elle est généralement plus élevée que celle des trois autres groupes.

Les zébus se caractérisent par une grande amplitude de variation de la température corporelle. Le matin, leur température est plus faible que celle des autres groupes; le soir, elle est plus élevée que celle des $1 / 2$ Montbéliard et des 1/4 Montbéliard, mais plus faible que celle des $1 / 2$ Charollais.

Nous avons indiqué plus haut que, pour les températures basses, les 1/4 Montbéliard se comportaient comme des I/2 Montbéliard; leur température est supérieure à celle des zébus et inférieure à celle des $1 / 2$ Montbéliard ct, pour les hautes températures, le sang zébu intervient; leur 'température est inférieure à celle des zébus, mais supérieure à celle des 1/2 Montbéliard.

Il semblerait à première vue que nos observations soient en contradiction avec les conclusions de Rhoad. Nous ne le pensons pas. Nous avons pris les 
températures le soir, alors que cel auteur les a enregistrées à 10 et 15 heures, et les résultats que nous avons obtenus avec le Heat-tolerance Test de Rhoad sont conformes à ses propres conclusions.

De plus, les conditions climatiques auxquelles étaient soumis nos animaux étaient sévères tout au long de l'année et les animaux étaient depuis longtemps acclimatés à ces conditions.

Leurs réactions diffèrent également de celles des animaux qui, occasionnellement, sont soumis à des tests de durée relativement réduite dans des chambres climatiques et qui, le reste de l'année, demeurent soumis à un climat tempéré. Notre observation est à rapprocher de celles faites par Mc Dowell et ses collaborateurs qui, étudiant la repeatability des tests de tolérance à la chaleur auxquels étaient soumis des animaux en chambre climatique, ont trouvé que les réponses aux tests dépendaient des températures auxquelles étaient soumis les animaux, dans la nature, entre les tests.

Nous pensons, comme Rhoad et Bonsma, que le zébu opere sa regulation thermique surtout par évaporation cutanée car en mars, avril et mai, alors que la température ambiante est élevée mais que le déficit de saturation est également élevé, la régulation thermique se fait facilement; mais lorsque le déficit de saturation est faible, même avec une température relativement faible, les perturbations de la température corporelle sont plus fortes.

Comme la surface de la peau des zébus, par unité de poids, est plus grande que chez les taurins, ce qui est un avantage quand l'animal doit disperser une forte quantité de chaleur, devient un inconvénient lorsqu'il doit lutter contre le refroidissement; ainsi peut-on expliquer l'hypothermie des zébus par rapport aux métis lorsque la température ambiante est basse.

Les mécanismes de la régulation thermique chez les taurins et les zébus doivent être différents. En effet, en Afrique tropicale, on note que les zébus vivent dans les zones à fortes temperatures et fort déficit de saturation, alors que les taurins des races N'Dama se répartissent dans les zones à températures relativement peu élevées, mais à faible déficit de saturation, et qu'ils s'acclimatent plus facilement que les zébus aux zones équatoriales à forte humidité.

Des essais en cours nous permettront peut-être de préciser les mécanismes intimes de la thermorégulation de ces animaux acclimatés aux zones torrides.

En résumé, il semble que les zébus, au lieu de dépenser de l'énergie à assurer à tout prix leur homéothermie, supportent sans en souffrir des variations de leur température beaucoup plus grandes que celles observées chez les métis Montbéliard.
Les métis Charollais étant, quant à eux, en hyperthermie permanente.

Pratiquement, les métis Montbéliard sont mieux adaptés aux climats du type soudanais que les métis Charollais; le poil bourru de ces derniers est certainement la cause de leur inadaptabilité.

Centre fédéral de Recherches zootechniques de l'A.O.F. Bamako (Soudan).

\section{VII. - BIBLIOGRAPHIE}

BISSCHOP (J.-H.-R.). - The Relation between Environment and Animal Breeding of Cattle in Semi-Arid Regions of South Africa. 13 th Internat. Vet. Conf. Rep., 2, p. 935-968.

BONSMA (J.-C.), - The Influence of Climatological Factors on Cattle. Observations on Cattle in Tropical Regions. Farm. in S. Africa, 1940. 15, p. 373-385.

BONSMA (J.-C.). - Influence of the Color and Coat Cover on Adaptability of Cattle. Farming in South Africa, 1943. 18, p. 101-120.

BONSMA (J.-C.). - Ecological Animal Husbandry Research and its Application in maintaining a Permanent Pastoral Industry. Proc. But. Comm. Sc. Off. Conf. Pretoria, 1949, Sect. A. Climatic Factor, I, 21.

BONSMA (J.-C.). - Breeding Cattle for increasing Adaptability to Tropical and Subtropical Environments. J. Agric. Sci., 1949, 39, p. 204-221.

BRODY (S.). - Environmental Physiology with Special Reference to Domestics Animals. I. Physiological backgrounds. Missouri Agri. Exp. Sta. Res. Bull., 1948, 423, 43 pages.

BRUYERE (P.). - De l'adaptation des bovins aux climats chauds. Bull. agric. Congo Belge, 1954,45 , p. 1057-1083.

DUHOTE (E.). - Les climats et l'organisme humain. 1948, 1 volume, 127 pages. Presses Universitaires, Paris.

FARINAUD (M.-E.). - L'homme et le climat tropical. Médecine tropicale, 1944, 3, p. 194-224.

FINDLAY (J.-D.). - The Effect of Temperature, Humidity, Air Movement and Solar Radiation on the Behaviour and Physiology of Cattle and other Farm Animals: Hannah Dairy Res. Instit., 1 volume, 178 pages.

FORBES (E.-B.), BRAHMANN (W.-W.), KRISS (M.), FRIES (S.-A.) et coll. - The Influence of the Environmental Temperature on Heat Production of Cattle. J. Agriculture, 1926, 33, p. $578-589$. 
FREBORN (S.-B.), REGAN (W.-M.) et BERRY (L.-J.). The Effect of Petroleum Oil Spray on Dairy Cattle. J. Econ. Entomology, 1934, p. 27.

GALAAS (R.-F.). - Effect of Atmospheric Temperature on Body Temperature and Respiration Rate of Jersey Cattle. Journ. Dairy Sci., 1945, 28, p. 555-563.

GALAAS (R.-F.) - - A Study on Heat Tolerance in Jersey Cows. Jour, Dairy SC., 1947, 30, p. 79-85.

HAMMOND (J.). -- Report on Cattle Breeding in Jamaica and Trinidad. Empire Marketing Board, 1932, 58.

HAMMOND (J). - Environmental Conditions and Livestock Breeding. Prob. Zivton, 1936, p. 101112.

HAMMIOND (J.).:- $\boldsymbol{A}$ Report on the Conditions of Animal Production in Australia. 1938. Counc. for Sc. and Ind. Res. of Australia. Pamphlet, $n^{0} 79,1$ volume, 24 pages.

JOSHI (N.-R.) et PHILLIPS (R.-W.), - Zebu Cattle of India and Pakistan. F.A.O. Agricult. St., no 19, 1 volume, 256 pages, Rome 1953.

KELLEY (R.-B.). - Zebu-Cross Cattle in Northern Australia. An Ecological Experiment. Commonwealth of Australia. Council for Sc. and Indust. Research, 1943, Bull. 173, 93 pages.

KIBLER (H.-H.), BRODY (S.) et WORSTELL (D.-M.), Influence of Temperature $50^{\circ}$ to $105^{\circ} \mathrm{F}$. on Heat Production and Cardiorespiratory Activities in Dairy Cattle. 1949, MO. Agr. Exp. Stat. Res. Bull., p. 435.

KIBLER (H.-H.) et BRODY (S.). - Influence of Tomperature $50^{\circ}$ to $5^{\circ} \mathrm{F}$ and $50^{\circ}$ to $95^{\circ} \mathrm{F}$ on Heat Production and Cardiorespiratory Activities of Dairy Cattle. 1949, Mo. Exp. Sta. Res. Bull., 450.

KIBLER (H.-H.) et BRODY (S.). -- Influence of Temperature $5^{\circ}$ To $95^{\circ} \mathrm{F}$ on Evaporative Cooling from the Respiratory and Exterior Body Surfaces. 1950. Missouri Exp. Stat. Res. Bull. 461.

KIBLER (H.-H.) et BRODY (S.). - Effect of Temperature $50^{\circ}$ to $105^{\circ} \mathbf{F}$ and $50^{\circ}$ to $9^{\circ} F$ on Heat Production and Cardiorespiratory Activities in Brahma, Jersey, Holstein Cows. 1950. Agr. Exp. Stat. Res. Bull., no 464.

KT.FIBER (M.) et REGAN (W.). - Influence of Temperature on Respiration of Cows. Proc. SOC. Exp. Biol. and Medecine, 1936, 33, p. 10-14.

LEE (Douglas-H.-K.). -.. Heat and Cold. Annual Review of Physio., 1948, 10, p. 365-386.
LEE (D.-H.-K.) et PHILLIPS (R.-W.). - Assessment of the Adaptability of Livestock to Climatic Stress. J. Anim. Sc., 1948, 7, p. 391-425.

Mc DOWELL (R.-E.), MATTHEWS (C.-A.) et LEE (D.-H.-K.). - Repeatability of an Experimental Heat Tolerance Test and the Influence of the Season. Jour. of. Anim. SC., 1953, 12, p. 757-764.

PAGOT (J.). - Climat et production de viande. Ann. de la Nutri. et de l'Alim., 1952, 6, p. 255-266.

PAGOT (J.). - Production laitière en zone tropicale. Rev. El. et Méd. Vét. Pays Tropicaux, 1951, 5, p. 173-190.

PAGOT (J.). - Croisement taurins-zébus. Etude biométrique des résultats obtenus à l'Office du Niger. Rev. El. et Méd. Vét. Pays Tropicaux, 1951, 5, p. 52-62.

PHILLIPS (R.-W.). - The Cattle of India. Heredity, 35, p. 273-288, 1944

PHILLIPS (R.-W.). - L'élevage en milieu défavorable. Étude agricole de la H.A.U., no 1 , 1949, Rome.

PHILLIPS (R.-W.), - L'amélioration du bétail dans les régions tropicales et subtropicales. 1950. F.A.O. Progrès et mise en valeur, cahier no 6 , 1 volume, 58 pages.

PIERY (M.) et coll. — Traité de climatologie biologique et médicale. Massoñ, 1934:

PILLE (G.). - Les échanges chlorurés en biologie tropicale. Accidents graves de déshydratation extracellulaire par carence chlorurée climatérique, observés à Fort-Lamy. Médecine tropicale, 1949, 9, p. 896-950.

PILLE (G.), 一Échanges chlorurés et climatométrie des zones intertropicales. Biol, médicale, 1953 , 42, p. 709-776

PILIE (G.). - Essai d'une politique alimentaire en Afrique noire. Conf. de. l'Élevage, Dakar, 1954. 13 pages polycopiées.

REGAN (M.-H.) et RICHARDSON (G.-A.). - Reaction of the Dairy Cows to Changes in Environmental Temperature. J. Dairy SC., 1938, 21, p. 73-79.

RHOAD (A.-O.). - The Influence of Environmental Temperature on the Respiration of Dairy Cattle in the Tropics. J. Agric. Sc., 1936, 26, p. 36-44.

RHOAD (A.-O.). - Some Observations on the Response of Purebred Bos taurus and Bos indicus Cattle and theix Cross-Bred Type to Certains Conditions of the Environment. Am. Soc. Anim. Prod., p. 284-285, 1939. 
RHOAD (A.-O.). - A Method of essaying Differences in Adaptability of Cattle to Tropical and Subtropical Climates. Emp: Jour. Exp. Agric., 1940, 8, p. 31.

RHOAD (A.-O.). - Climate and Livestock Production. Climate and Man. Yearbook of Agriculture U.S.D.A., p. 508-516, 1941.

RHOAD (A.-O.). - Water Expenditure in Bos taurus and Bos indicus in relation to Adaptability to a Tropical Environment. Proc. 8th. American Scientific. cong., 1942, p. 115-123.

RHOAD (A.-O.). - A Scale of Heat Tolerance for Cattle. Journ. Anim. Sci., 1942, 1, p. 85.

RHOAD (A.-O.) et BT.ACK (W.-H.). - Hybrid Beef Cattle for Subtropical Climates. 1943. Cir. 673 U.S.D.A., Washington D.C.

RHOAD (A,-O.). - The Iberia Heat Tolerance Test of Cattle. Tropic. Agric., 1944, 9, p. 162-164.

RITZMAN (E.) et BENEDICT (F.-G.). - Nutritional Physiology of the Adult Ruminant. Carnegie Institution of Washington, 1938.

ROUCH. - L'atmosphère et la prévision du temps. 1 volume, Armand Colin, Paris.

SANSON (J.): - Climatologie appliquée. Publica- tion de la Météorologie nationale. 1949. 1 volume, 210 pages. Édit. Blondel la Rougery, Paris.

SEATH (D.-M.) et MILLER (G.-D.). - The Relative Importance of High Temperature and High Humidity as Factor influencing Respiration Rate Body Temperature and Pulse Rate of Dairy Cows. Journ. Dairy Sc., 29, p. 465-472, 1946.

SEATH (D.-M.) and MLLER. - Effect of Warm Weather on Grazing Performance of Milking Cows. Jour. Dairy Sc., 29, p. 199-206, 1946.

SEATH-MILLER. - Heat Tolerance Comparisons between Jersey and Holstein Cows. Jour. of. Anim. Sc., 6, 1 février 1947, p. 24-33.

SEATH (D.-M.). - Heritability of Heat Tolerance in Dairy Cattle. Jour. Dairy SC., 1947, 30, p. 136144.

SEATH (D.-M.) et MILLER (G.-D.). - Effect of Shade and Sprinkling, with Water on Summer Confort of Jersey Cows. Journ. Dairy Sc, ; 1947, 30, p. 255-261.

SLMONET (R.), - La chaleur. 1947. Un volume. Presses Universitaires. Paris.

VEYSSEREAU (A.). - Méthodes statistiques en biologie et en agronomie. 1948. 1 volume, 381 pages. J.-B. Baillère, Paris.

\section{SUMMARY}

The Sotuba Livestock Expcrimental Station near Bamako (French Sudan) carried out cxporiments on a herd consisting mainly of Peul pure zebu cattle, $1 / 2$ zebu $\times$ charollais and zebu $\times$ Montbéliard crossed breeds, as well as of a few $3 / 4$ zebu $\times 1 / 4$ Montbéliard long adapted to the local environment.

The purpose of the experiments was to assess the influence of the French Sudanese climate on the body temperature of each group of these animals.

The greatest variation in body temperature was observed in Peul pure zebu cattle.

The research carried out on the reactions of the animals to heat differs from those obtained by other research workers who temporarily subjected animais which normally live in a temperate climate to short duration tests in psychrometric chamber.

The Sotuba experiments confirmed that the thermal regulation of zebus was achieved by transpiration through the skin.

The article contains many charts showing temperature variations according to the breeds.

\section{RESUMEN}

El centro de investigaciones zootécnicas de Sotuba, cerca de Bamako (Sudán Francés)idispone para sus trabajos de un rebaño integrado especialmente por cebìs de raza peul, cebus mestizos $\times$ charollais, cebús $\times$ montbéliard y algunos cebus $3 / 4 \times$ Montbéliard $1 / 4$, aclimatados ya hace largo tiempo a las condiciones del medio ambiente.

Parece interesante determinar en que medida los factores meteorológicos propios del clima sudanés eran susceptibles de influenciar la temperatura corporal individual de semejante grupo de animales. 
El estudio de las reacciones de los animales al calor demuestra que estas reacciones son distintas dé las obtenidas por diversos autores en animales sometidos ocasionalmente a pruebas de duración reducida en cámaras climaticas y que entre varias pruebas quedan sometidos a los efectos de un clima templado. cutánea.

Las experiencias han permitido confirmar que el cebú opera su regulación térmica por evaporación Numerosas tablas mustran las variaciones según les razas experimentadas. 
Tableau no $n^{\circ}$. - CARACTÉRISTIQUES DU CLIMAT DE LA STATION EN 1950 (SEGOU)

\begin{tabular}{|c|c|c|c|c|c|c|c|c|c|c|c|c|c|}
\hline & JANVIER & FÉVRIER & MARS & AVRIL & MAI & JUIN & JULLLET & AOUT & SEPTEM. & OCTOBRE & NOVEM. & DECEM. & ANNÉEE \\
\hline \multicolumn{14}{|l|}{ Température. } \\
\hline Temperature maxima moyenne & 31,8 & 34,3 & 37,2 & 39,8 & 39,3 & 36,1 & 31,9 & 29 & 31 & 32,8 & 34,2 & 32,5 & 34,15 \\
\hline Température minima moyenne & 15 & 17 & 20,7 & 24,5 & 25,8 & 24,3 & 22,8 & 22,1 & 22,2 & 21,5 & 17,9 & 15,5 & 20,77 \\
\hline $\begin{array}{l}\text { Écart entre les muyenines pré- } \\
\text { cédentes } \ldots \ldots \ldots \ldots \ldots \ldots\end{array}$ & 16,8 & 17,3 & 16,5 & 15,3 & 13,5 & 11,8 & 9,1 & 6,9 & 8,8 & 11,3 & 16,3 & 17 & \\
\hline I'empérature maxima .......... & 36,2 & 39,3 & 40,5 & 42,7 & 41,8 & 40,2 & 38,5 & 32,1 & 33,8 & 35,7 & 36,6 & 35,7 & \\
\hline Date $\ldots \ldots \ldots \ldots \ldots \ldots$ & 28 & 18 & 29 & 8 & 20 & 4 & 2 & 14 & 25 & 25 & 9 & 17 & \\
\hline Température minima $\ldots . . .$. & 11,8 & 13,8 & 12,8 & 19,5 & 22,5 & 20,8 & $-19,5$ & 20,1 & 20,2 & 19,4 & 11,0 & 11,6 & \\
\hline Date $\ldots \ldots \ldots \ldots \ldots \ldots \ldots$ & 14 & 10 & 10 & 6 & 7 & 30 & 17 & 15 & 7 & 30 & 30 & 31 & \\
\hline I'empérature moyenne du matin. & 17,4 & 19,0 & 23,1 & 26,1 & 27,1 & 25,9 & 23,9 & 23,0 & 23,2 & 22,4 & 19,6 & 17,3 & \\
\hline Température moyenne du soir. . & 29,8 & 31,8 & 35,3 & 37,0 & 37,5 & 34,4 & 29,3 & 27,8 & 27,8 & 29,5 & 29,5 & 28,5 & \\
\hline \multicolumn{14}{|l|}{ Pluie. } \\
\hline Précipitations totales mensucllos, & 0 & & 1,2 & 55,5 & 23.5 & 82,7 & 218,8 & 336,5 & 126,4 & 54,0 & 0 & 0.2 & 899,1 \\
\hline Nombre de jours de pluie...... & 0 & & 1 & 3 & 6 & 0 & 11 & 19 & 14 & 5 & 0 & 0 & 08 \\
\hline $\begin{array}{l}\text { Précipitations moyennes des } \\
\text { années précédentes .......... }\end{array}$ & 0,1 & 0,1 & 1,0 & 15,0 & 24,1 & 43 & 182,4 & 243,3 & 121,8 & 22 & 3,2 & 0 & \\
\hline \multicolumn{14}{|l|}{ Vent. } \\
\hline Direction & $E$ & E & $E$ & $\mathrm{NE}$ & E & W & SW & WSW & WSW & Calme & $\mathrm{NE}$ & $\mathrm{NE}$ & \\
\hline Vitesse $\ldots \ldots \ldots \ldots \ldots \ldots$ & 4 & 4 & 3 & 3 & 3 & 3 & 3 & 3 & 2 & & 3 & 3 & \\
\hline Tension vapeur à $6 \mathrm{~h} \ldots \ldots \ldots$ & 9,4 & 8,2 & 8,4 & 15,9 & 21,8 & 24,2 & 25,7 & 26,2 & 26,5 & 24,7 & 16,0 & 10,8 & 18,15 \\
\hline à $18 \mathrm{~h} \ldots \ldots \ldots$ & 9,3 & 7,2 & 8,2 & 12,6 & 15,9 & 21,4 & 25,2 & 28,0 & 27,4 & 26,9 & 15,4 & 10,5 & \\
\hline Humidité en $\% 6 \mathrm{~h}, \ldots, \ldots \ldots$ & 49 & 38 & 30 & 46 & 62 & 73 & 86 & 93 & 93. & 90 & 71 & 56 & \\
\hline $12 \mathrm{~h}$ & 23 & 14 & 13 & 25 & 34 & 47 & 65 & 79 & 69 & 57 & 27 & 22 & \\
\hline $18 \mathrm{~h}, \ldots \ldots \ldots$ & 24 & 17 & 14 & 20 & 26 & 40 & 63 & 78 & 74 & 65 & 37 & 27 & \\
\hline \multicolumn{14}{|l|}{ Ciel. Nébulosité : } \\
\hline inférieure à $218 \ldots \ldots$ & 6 & $6^{\cdots}$ & $6^{-}$ & 3 & 0 & I $\cdots$ & -0 & 0 & 0 & 2 & 1 & 9 & \\
\hline supérieure à $2 / 8 \ldots \ldots \ldots$ & 1 & 0 & 0 & 1 & 3 & 1 & 8 & 8 & 5 & 2 & 3 & 0 & \\
\hline Évaporation en $m m \ldots \ldots \ldots$ & 269,6 & 345,6 & 479,9 & 484,1 & 371,0 & 273,2 & $-166,3$ & 74,0 & 86,6 & 118,3 & 217,9 & 256,2 & \\
\hline
\end{tabular}



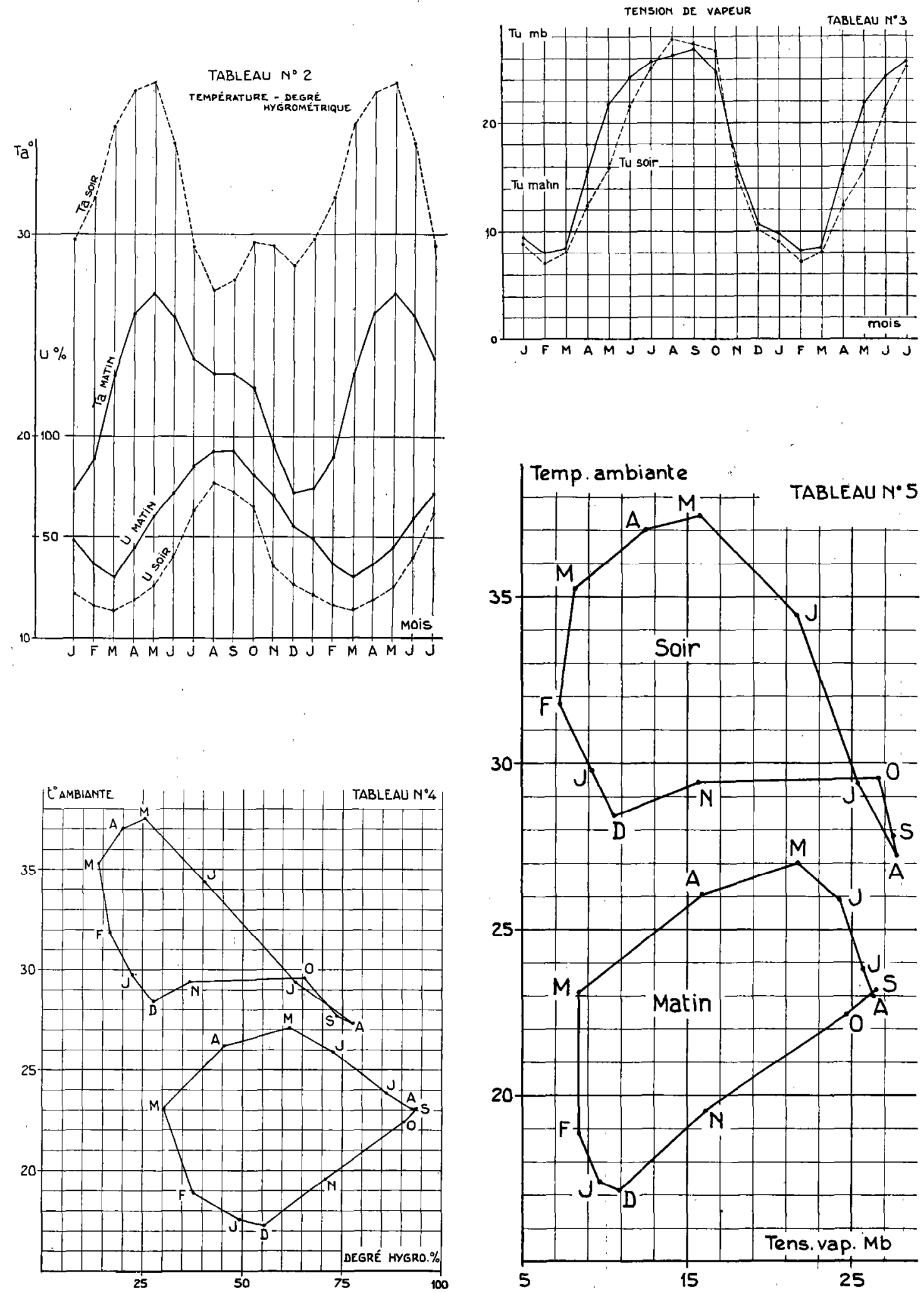
Tableau $n^{\circ}$ 6. - OBSERVATIONS DU MATIN

\begin{tabular}{|c|c|c|c|c|c|c|c|}
\hline \multirow{2}{*}{ MOIS } & \multirow{2}{*}{$\begin{array}{l}\text { TEMPÉR. } \\
\text { ambiante }\end{array}$} & \multirow{2}{*}{$\begin{array}{l}\text { HUMIDITÉ } \\
\text { relative }\end{array}$} & \multirow{2}{*}{$\begin{array}{c}\text { TENSION } \\
\text { de vapeur Mb } \\
\text { et } 1,10\end{array}$} & \multicolumn{4}{|c|}{ TEMPÉRATURE CORPORELLE } \\
\hline & & & & $\begin{array}{c}1.2 \\
\text { Charollais }\end{array}$ & $\begin{array}{c}1 / 2 \\
\text { Montbéliard }\end{array}$ & $\begin{array}{c}1 / 4 \\
\text { Montbéliard }\end{array}$ & Zébus \\
\hline Janvier... . . . . . . & 17,46 & 49,2 & 9,77 & 38,08 & 38,08 & 38,07 & 38,06 \\
\hline Février ........... & 18,96 & 37,8 & 8,21 & 38,19 & 38,19 & 38,10 & 38,12 \\
\hline Mars... . . . . & 23,07 & 30,1 & 8,41 & 38,22 & 38,26 & 38,09 & 38,19 \\
\hline Avril . . . . . . . & 26,12 & 45,6 & 15,85 & 38,20 & 38.23 & 38,15 & $38 ; 18$ \\
\hline Mai . . & 27,08 & 61,9 & 21,79 & 38,26 & 38,22 & 38,28 & 38,19 \\
\hline Juin.... & 25,94 & 72,7 & 24,19 & 38,27 & 38,18 & 38,19 & $38 ; 18$ \\
\hline Juillet . & 23,85 & 86,2 & 25,65 & 38,17 & 38,16 & 38,17 & 38,19 \\
\hline Août & 23,02 & 92,8 & 26,61 & 38,16 & 38,13 & 38,11 & 38,14 \\
\hline Septembre & 23,15 & 92,9 & 26,46 & 38,20 & 38,15 & 38,14 & 38,15 \\
\hline Octobre.... & 22,44 & 90,3 & 24,72 & 38,14 & 38,13 & 38,14 & 38,18 \\
\hline Novembre & 19,60 & 71 & 16,01 & 38,12 & 38,04 & 38,08 & 38,07 \\
\hline Décembre......... & 17,25 & 55,5 & 10,76 & 38,08 & 38,04 & 38,05 & 38,04 \\
\hline
\end{tabular}

Tableau n० 7 . - OBSERVATIONS DU SOIR

\begin{tabular}{|c|c|c|c|c|c|c|c|}
\hline \multirow{2}{*}{ MOIS } & \multirow{2}{*}{$\begin{array}{l}\text { TEMPÉR. } \\
\text { ambiante }\end{array}$} & \multirow{2}{*}{$\begin{array}{l}\text { HUMMDITÉ } \\
\text { relative }\end{array}$} & \multirow{2}{*}{$\begin{array}{c}\text { TENSION } \\
\text { de vapeur Mb } \\
\text { et } 1 \cdot 10\end{array}$} & \multicolumn{4}{|c|}{ TEMPÉRATURE CORPORELLE } \\
\hline & & & & $\begin{array}{c}1.2 \\
\text { Charollais }\end{array}$ & $\begin{array}{c}1 / 2 \\
\text { Montbéliard }\end{array}$ & $\begin{array}{c}1 / 4 \\
\text { Montbéliard }\end{array}$ & Zébus \\
\hline Janvier.. & 29.78 & 22,5 & 9,34 & 38,78 & 38,78 & 38,76 & 38,72 \\
\hline Février $\ldots \ldots \ldots \ldots$ & 31,81 & 16,6 & 7,16 & 38,59 & 38,59 & 38,52 & 38,56 \\
\hline Mars.... & 35,33 & 14,3 & 8,25 & 38,68 & 38,71 & 38,67 & 38,68 \\
\hline Avril. & 37,01 & 19,9 & 12,60 & 38,75 & 38,80 & 38,66 & 38,68 \\
\hline Mai ... & 37,5 & 25,7 & 15,91 & 38,88 & 38,70 & 38,68 & 38,63 \\
\hline Juin... . & 34,41 & 40,1 & 21,38 & 39,07 & 38,75 & 38,79 & 38,74 \\
\hline Juillet $\ldots \ldots \ldots \ldots \ldots$ & 29,47 & 63,4 & 25.21 & 38,97 & 38,81 & 38,88 & 38,94 \\
\hline Août ... & 27,23 & 77,8 & 27,95 & 38,95 & 38,84 & 38,82 & 38,83 \\
\hline Septembre ....... & 27,81 & 73,5 & 27,44 & 39,53 & 39,02 & 39,10 & 39,10 \\
\hline Octobre.... & 29,54 & 65,3 & 26,88 & 39,36 & 39,04 & 39,08 & 39,16 \\
\hline Novembre .. & 29,45 & 36,8 & 15,40 & 39,29 & 39,02 & 39,03 & 39,03 \\
\hline Décembre.... & 28,47 & 27,4 & 10,55 & 39,03 & 38,79 & 38,87 & 38,77 \\
\hline
\end{tabular}



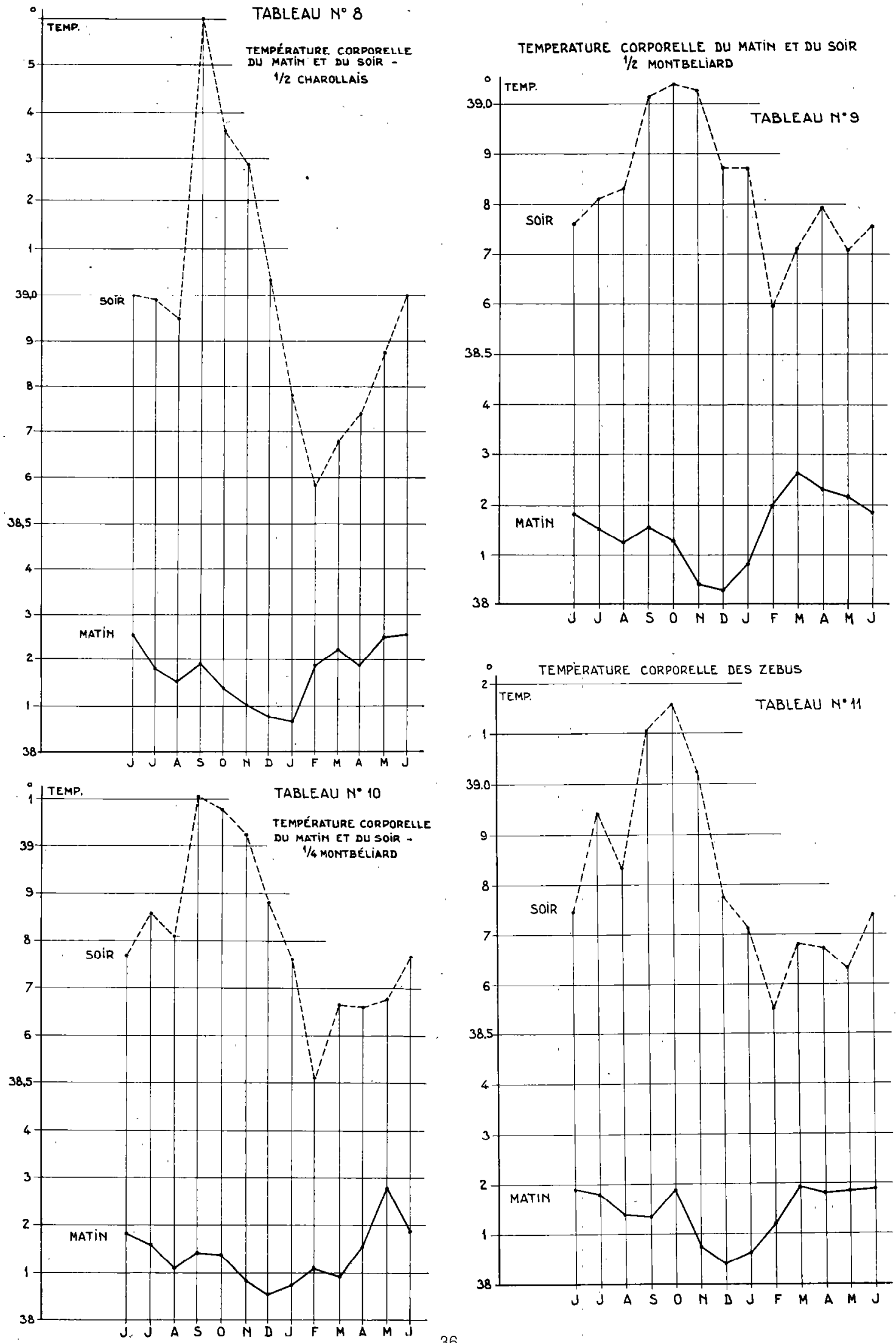

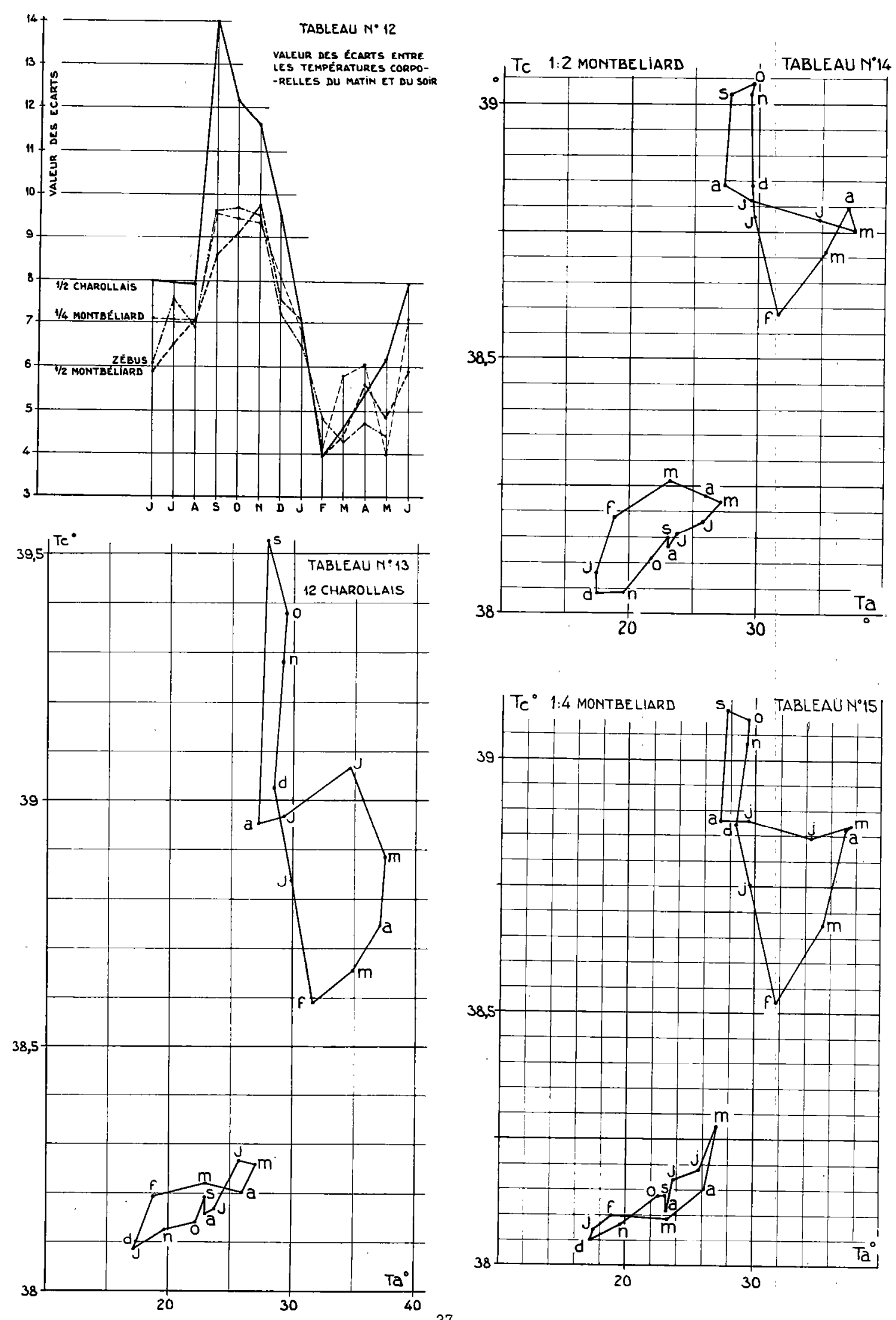

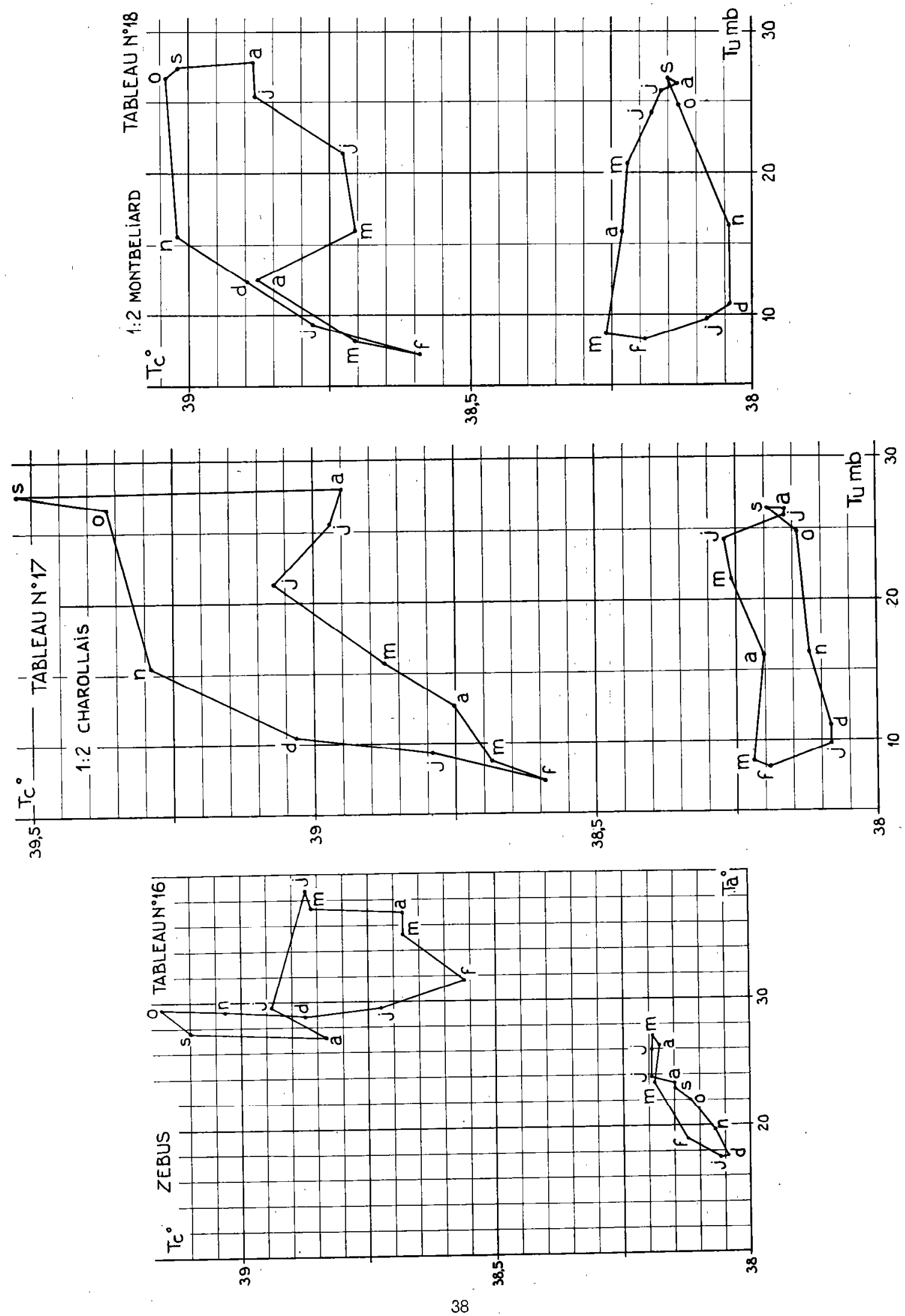

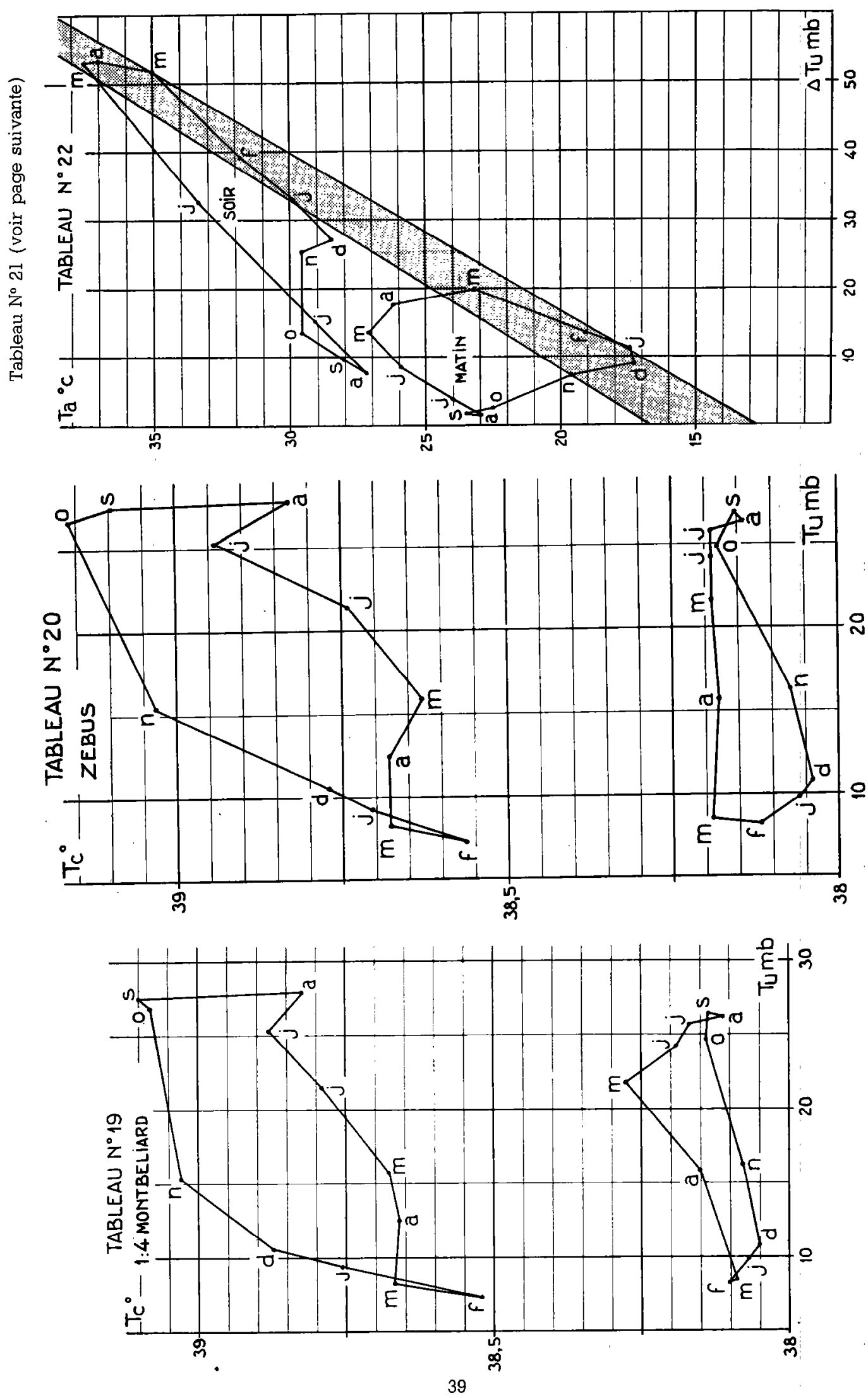
TABLEAU $n^{\circ} 21$

\begin{tabular}{|c|c|c|c|c|c|c|}
\hline & JANVIER & FÉVRIER & MARS & AVRIL & MAI & JUIN \\
\hline \multicolumn{7}{|l|}{ Observations du matin } \\
\hline Température ambiante $\ldots \ldots \ldots \ldots \ldots \ldots \ldots$ & 17,4 & 19,0 & 23,1 & 26,1 & 27,1 & 25,9 \\
\hline $\begin{array}{l}\text { Tension maximum de la vapeur d'eau à la tempé- } \\
\text { raturc obscrvćc } \ldots \ldots \ldots \ldots \ldots \ldots \ldots \ldots \ldots \ldots \ldots\end{array}$ & 19,8 & 21,8 & 28,0 & 33,5 & 35,6 & 32,6 \\
\hline Tension de vapeur observée ........ & 9,4 & 8,2 & 8,4 & 15,9 & 21,8 & 24,2 \\
\hline Déficit de saturation $\ldots \ldots \ldots \ldots \ldots \ldots$ & 10,4 & 13,6 & 19,6 & 17,6 & 13,8 & 8,4 \\
\hline Observations du soir & & ' & & a & & \\
\hline Température ambiante $\ldots \ldots \ldots \ldots \ldots \ldots \ldots \ldots$ & 29,8 & $31 ; 8^{\circ}$ & 35,3 & 37,0 & 37,5 & 34,4 \\
\hline $\begin{array}{l}\text { Tension maximum de la vapeur d'eau à la tempé- } \\
\text { rature observée } \ldots \ldots \ldots \ldots \ldots \ldots \ldots \ldots \ldots \ldots \ldots \ldots\end{array}$ & 41,6 & 46,6 & 58,2 & 62,4 & 64,0 & 54,0 \\
\hline Tension de vapeur observée ........... & 9,3 & 7,2 & 8,2 & 12,6 & 15,9 & 21,4 \\
\hline Déficit de saturation. . & 32,3 & 39,4 & 50,0 & 49,8 & 49,1 & 32,6 \\
\hline & JUILLET & AOUT & STEPTEM. & OCTOBRE & NOVEM & DÉCEM. \\
\hline \multicolumn{7}{|l|}{ Observations du matin } \\
\hline Température ambiante $\ldots \ldots \ldots \ldots \ldots \ldots$ & 23,9 & 23,0 & 23,2 & $.22,4$ & 19,6 & 17,3 \\
\hline $\begin{array}{l}\text { Tension maximum de la vapeur d'eau à la tempé- } \\
\text { rature observée } \ldots \ldots \ldots \ldots \ldots \ldots \ldots \ldots \ldots \ldots \ldots \ldots\end{array}$ & 29,4 & 27,8 & 28,3 & 26,8 & 22,8 & 19,6 \\
\hline Tension de vapeur observée $\ldots . . . . . \ldots \ldots \ldots \ldots$ & 25,7 & 26,2 & 26,5 & 24,7 & 16,0 & 16,8 \\
\hline $\begin{array}{c}\text { Déficit de saturation................... } \\
\text { Observations du soir }\end{array}$ & 3,7 & 1,6 & 1,8 & 2,1 & 6,8 & 8,8 \\
\hline Température ambiante $\ldots \ldots \ldots \ldots \ldots \ldots \ldots$ & 29,5 & 27,2 & 27,8 & 29,5 & 29,5 & 28,5 \\
\hline $\begin{array}{l}\text { Tension maximum de la vapeur d'eau à la tempé- } \\
\text { rature observée } \ldots \ldots \ldots \ldots \ldots \ldots \ldots \ldots \ldots \ldots \ldots\end{array}$ & 40,9 & 35,9 & 37,0 & 40,9 & 40,9 & 38,6 \\
\hline Tension de vapeur observée ... & 25,2 & 28,0 & 27,4 & 26,9 & 15,4 & 10,5 \\
\hline Déficit de saturation. . & 14,7 & 7,9 & 9,6 & 14,0 & 25,5 & 27,1 \\
\hline
\end{tabular}



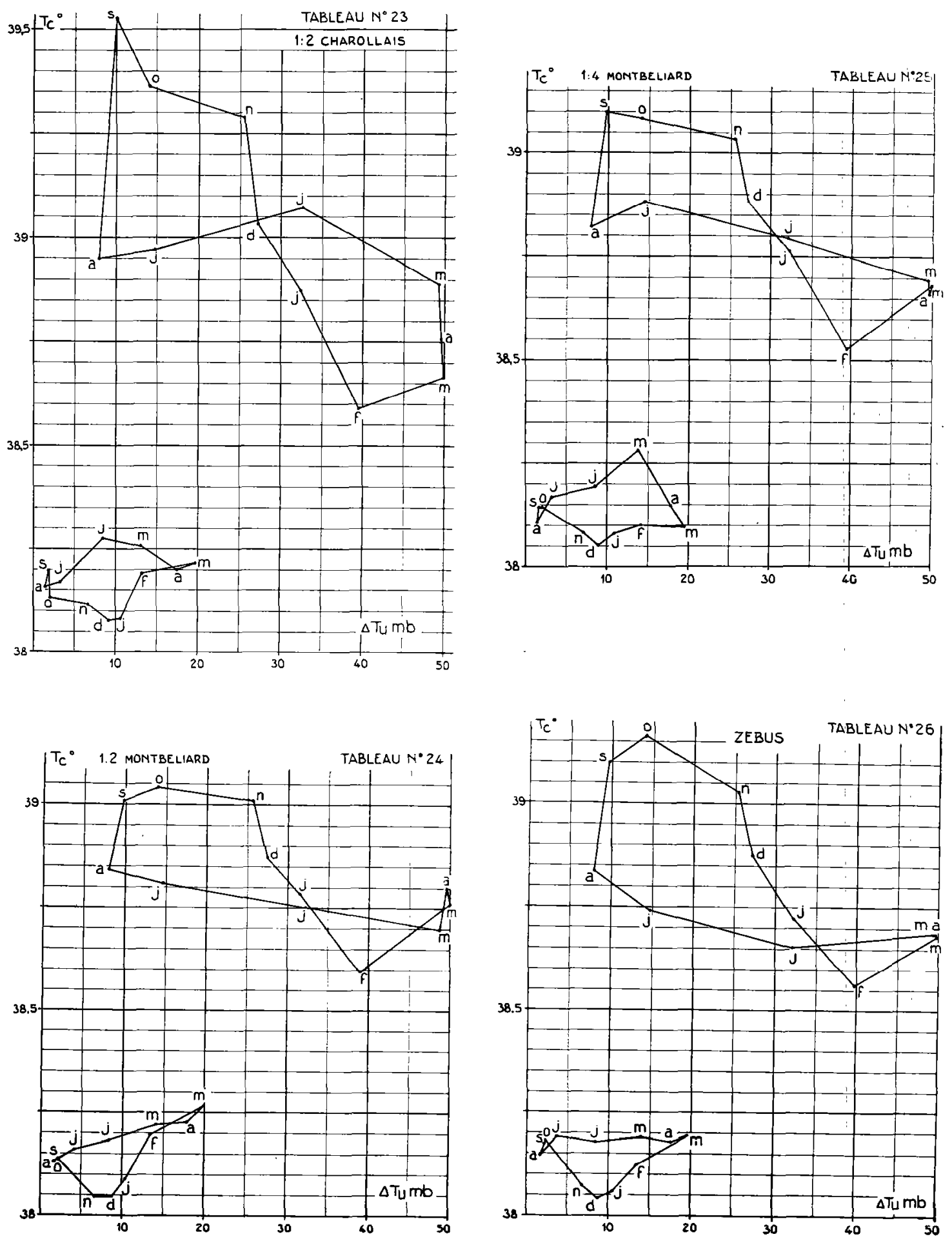